\title{
Fractional Scaling of Magnetic Coercivity in Electrical Steels
}

\author{
M. NAJGeBAUER* \\ Faculty of Electrical Engineering, Częstochowa University of Technology, \\ al. Armii Krajowej 17, 42-200 Częstochowa, Poland
}

\begin{abstract}
The paper presents an application of the fractional scaling procedure in the analysis of magnetic coercivity. The frequency and excitation dependences of measured coercivity can be expressed in a single curve using properly scaled coercivity and frequency values. The scaling parameters will be presented for three different electrical steels.

DOI: 10.12693/APhysPolA.131.633

PACS/topics: 75.50.Bb, 75.60.Ej, 89.75.Da
\end{abstract}

\section{Introduction}

The scaling hypothesis was first postulated by Widom based on phenomenological grounds. It provides data collapse and a relation between critical exponents, which allows one to reduce the number of independent parameters characterizing the critical behavior $[1,2]$.

Scaling and data collapse are usually used to analyze critical phenomena and phase transitions. However, they can also be applied to analyze phenomena far from the critical point, e.g. related to magnetization phenomena as hysteresis loop [3-8] or magnetic viscosity $[9,10]$.

The Widom scaling has been successfully applied in analysis of power losses for various types of soft magnetic materials [11-15]. The Widom procedure was also used in scaling of magnetic coercivity [16]. However, the results of the coercivity scaling were not satisfactory. The modified procedure for the coercivity scaling using the hyperbolic tangent transformation was proposed in [17]. Although this procedure improved the coercivity scaling, it also introduced a large number of new parameters to be estimated.

In the paper, a new approach to the scaling of magnetic coercivity, based on a fractional procedure, is presented. The proposed coercivity scaling is examined for electrical steels with different internal structures as non-oriented, grain-oriented and microcrystalline ones.

\section{Fractional scaling of magnetic coercivity}

In general, the Widom scaling assumes that a functional relation $F=f\left(x_{1}, x_{2}, \ldots, x_{n}\right)$ describing the phenomenon is a general homogeneous function, which is defined as:

$$
\begin{aligned}
& \exists a_{1 \ldots n}, p: \forall \lambda>0 \\
& \lambda^{p} f\left(x_{1}, x_{2}, \ldots, x_{n}\right)=f\left(\lambda^{a_{1}} x_{1}, \lambda^{a_{2}} x_{2}, \ldots, \lambda^{a_{n}} x_{n}\right),(1)
\end{aligned}
$$

where $x_{1} \ldots x_{n}$ - variables, $a_{1} \ldots a_{n}$ and $p-$ scaling exponents.

\footnotetext{
*corresponding author; e-mail: najgebauer@el.pcz.czest.pl
}

The present study assumes that magnetic coercivity depends on maximum magnetic induction $B_{m}$ and frequency of magnetizing field $f$, which yields the following functional relation $H_{C}=h\left(f, B_{m}\right)$. According to (1), this relation may be written

$$
\exists c, d, e: \forall \lambda>0 \lambda^{d} H_{C}=h\left(\lambda^{e} f, \lambda^{c} B_{m}\right) .
$$

For the particular choice $\lambda=B_{m}^{-1 / c}$, the relation (2) may be transformed

$$
\begin{aligned}
& B_{m}^{-d / c} H_{C}=h\left(B_{m}^{-e / c} f, 1\right), \\
& B_{m}^{-\delta} H_{C}=h\left(B_{m}^{-\varepsilon} f, 1\right), \\
& H_{B}=h\left(f_{B}, 1\right)
\end{aligned}
$$

and finally reduced to the scaled form

$$
H_{B}=H\left(f_{B}\right),
$$

where $\delta, \varepsilon$ are scaling exponents, $H_{B}, f_{B}$ are scaled coercivity and frequency, while $H\left(f_{B}\right)=h\left(f_{B}, 1\right)$ is a scaling function.

In the classical scaling the right side of (3) is represented by the Maclaurin series, while in the fractional procedure - the variable $f_{B}$ is additionally substituted by $f_{B}^{x}$. It yields a fractional scaling formula for magnetic coercivity

$$
H_{B}=H_{0}+H_{1} f_{B}^{x}+H_{2} f_{B}^{2 x}+H_{3} f_{B}^{3 x}+\ldots,
$$

where $H_{0,1,2, \ldots}$ are scaling factors, $x$ is a fractional exponent.

The fractional scaling procedure has been previously examined for power losses in electrical steels [18] and magnetocaloric alloys [19]. It was proved that the simplest scaling formula (a single power law) gave the scaling results comparable to the use of more complex formulae [18]. For this reason, a reduced form of the formula (4) was chosen for the scaling of magnetic coercivity

$$
H_{B}=H_{0}+H_{1} f_{B}^{x} \text {. }
$$

\section{Results and discussion}

The fractional scaling formula of magnetic coercivity (5) is examined for commercially available electrical steels with non-oriented, grain-oriented and microcrystalline internal structure. The scaling exponents $\delta, \varepsilon$, $x$ and factors $H_{0,1}$ should be estimated from experimental data, containing a family of frequency dependences of 
magnetic coercivity, measured at various levels of magnetic induction. The exemplary measurements of magnetic coercivity, carried out using the computer-aided measuring system MAG-RJJ-2.0, are depicted in Fig. 1.

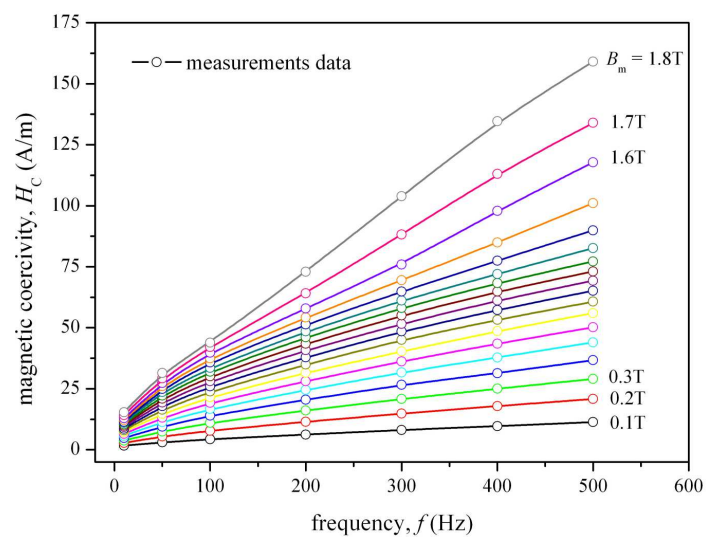

Fig. 1. Magnetic coercivity for grain-oriented electrical steel, 111-35-N5 grade.

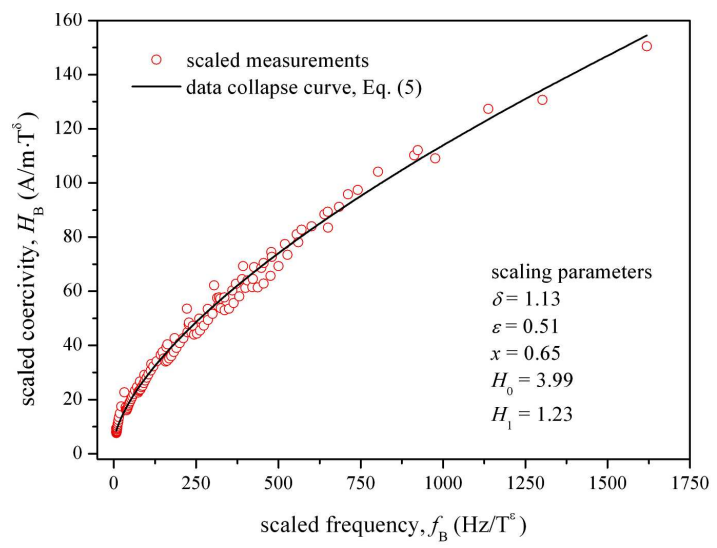

Fig. 2. Scaling and data collapse of magnetic coercivity for grain-oriented electrical steel, 111-35-N5 grade.

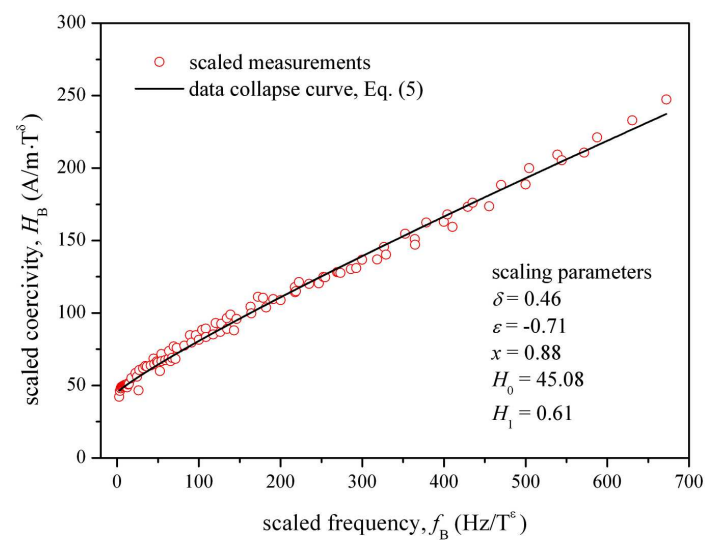

Fig. 3. Scaling and data collapse of magnetic coercivity for non-oriented electrical steel, M350-50A grade.

For the proper estimation of the parameters, all measured curves (points) of magnetic coercivity should be

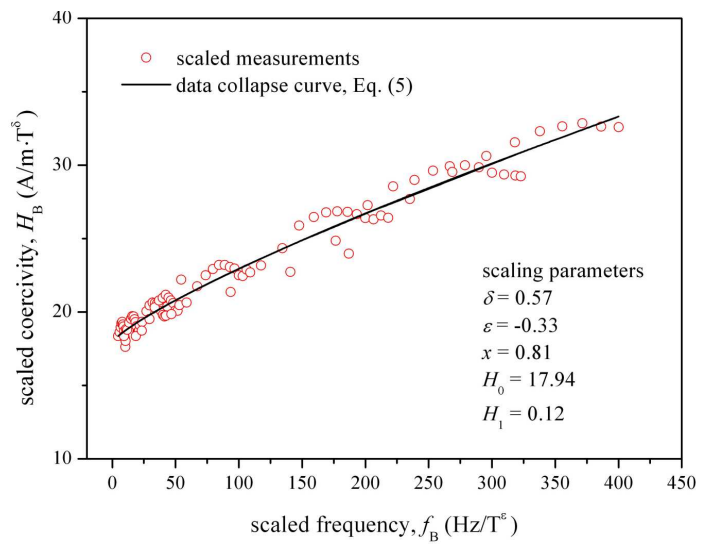

Fig. 4. Scaling and data collapse of magnetic coercivity for $6.5 \% \mathrm{Si}-\mathrm{Fe}$ steel, JNEX core.

collapsed onto a single curve. The results of the coercivity scaling using the fractional procedure are depicted in Figs. 2-4. Data collapse of coercivity measurements is obtained for all samples. However, in the case of $6.5 \% \mathrm{Si}-$ Fe steel some deviations of measurements from the data collapse curve are observed. It might be caused by lower number of data points used in the parameters estimation, comparing to the other samples. In order to evaluate the quality of the coercivity scaling, the coefficient of determination $R^{2}$ was calculated. The obtained values of $R^{2}$ are comparable and equal to $0.984,0.991$ and 0.972 for the samples of GO steel, NO steel and $6.5 \%$ steel, respectively. It confirms that the fractional procedure provides satisfactory results of the coercivity scaling for all examined steels. The presented results might be considered as verification of the scaling behavior of magnetic coercivity in electrical steels.

Finally, it should be noted that the scaling formula for magnetic coercivity (5) has a structure similar to the known frequency dependence of magnetic coercivity

$$
H_{C}=H_{C}(0)+B\left(H_{0} f\right)^{1 / n},
$$

where $H_{0}$ is a field amplitude, $B$ is a coefficient depending on material intrinsic parameters and $n$ is a coefficient ranging from 1 to $3[20,21]$. Moreover, the estimated values of the fractional exponent $x$ vary from 0.65 to 0.88 , thus they are in the range of the frequency exponent $1 / n$, defined in (6). It additionally confirms that the fractional scaling can be used in modeling of magnetic coercivity.

\section{Conclusions}

In the paper, the fractional scaling procedure was applied in the analysis of magnetic coercivity. It provides a simply formula for the scaled coercivity, similar to currently used models. This procedure was examined for commercially available electrical steels. For each sample, a family of measured coercivity dependences was collapsed onto a single curve. The results confirm the usefulness of the fractional scaling as well as prove the scaling behavior of magnetic coercivity in electrical steels. The results indicate the potential use of the fractional scaling 
in coercivity modeling for different types of soft magnetic materials. However, this assumption requires further research.

\section{References}

[1] B. Widom, J. Chem. Phys. 43, 3898 (1965).

[2] H.E. Stanley, Rev. Mod. Phys. 71, S358 (1999).

[3] A. Adedoyin, P. Andrei, IEEE Trans. Magn. 44, 3165 (2008).

[4] S. Takahashi, S. Kobayashi, T. Shishido, J. Phys. Condens. Matter 20, 035217/1 (2007).

[5] S. Kobayashi, N. Kikuchi, S. Takahashi, H. Kikuchi, Y. Kamada, J. Magn. Magn. Mater. 322, 1515 (2010).

[6] S. Kobayashi, S. Tsukidate, Y. Kamada, H. Kikuchi, T. Ohtani, J. Magn. Magn. Mater. 324, 215 (2012).

[7] S. Kobayashi, S. Takahashi, Y. Ishibashi, IEEE Trans. Magn. 48, 1449 (2012).

[8] L.K. Varga, J. Kováč, Acta Phys. Pol. A 126, 156 (2014).

[9] I.D. Mayergoyz, A.A. Adly, C. Korman, M. Huang, C. Krafft, J. Appl. Phys. 85, 4358 (1999).

[10] I.D. Mayergoyz, A.A. Adly, M.W. Huang, C. Krafft, IEEE Trans. Magn. 36, 3208 (2000).
[11] K. Sokalski, J. Szczygłowski, M. Najgebauer, W. Wilczyński, COMPEL 26, 640 (2007).

[12] M. Najgebauer, Prz. Elektrotechniczny 84, 213 (2008) (in Polish).

[13] K. Sokalski, J. Szczygłowski, Acta Phys. Pol. A 115, 920 (2009).

[14] K. Sokalski, J. Szczygłowski, Acta Phys. Pol. A 117, 497 (2010).

[15] J. Szczygłowski, P. Kopciuszewski, K. Chwastek, M. Najgebauer, W. Wilczyński, Prz. Elektrotechniczny 86, 45 (2010) (in Polish).

[16] M. Najgebauer, Solid State Phenom. 220-221, 646 (2015).

[17] M. Najgebauer, K. Sokalski, J. Szczygłowski, Archiv. Electr. Eng. 64, 351 (2015).

[18] M. Najgebauer, Acta Phys. Pol. A 128, 107 (2015).

[19] R. Gozdur, M. Najgebauer, J. Electr. Eng. 66, 37 (2015).

[20] A. Zhukov, M. Vázquez, J. Velázquez, C. García, R. Valenzuela, B. Ponomarev, Mater. Sci. Eng. A 226-228, 753 (1997).

[21] R. Grössinger, N. Mehboob, D. Suess, R.S. Turtelli, M. Kriegisch, IEEE Trans. Magn. 48, 3076 (2012). 